\title{
Research on the Protection Scheme of DG Interconnection Transition Mode Based on Decoupling Zero Sequence
}

\author{
Xia Lin ${ }^{1}$, Yuping $\mathrm{Lu}^{2}$, Yao Li ${ }^{1}$, Guibin $\mathrm{Zou}^{3}$, \\ 1. Shandong Zaozhuang Power Supply Company, Zaozhuang, China; \\ 2.Department of Electrical Engineering, Southeast University, Nanjing, China \\ 3. School of Electrical Engineering, Shandong University, Ji'nan, China \\ boulevard@126.com, pyliyao@sina.com, guibinzou@sdu.edu.cn
}

\begin{abstract}
More and more DG is connected with the grid through the low voltage distribution network, how to choose a protection scheme without depending on the nature of $D G$, and with reliable operation mode is a big issue encountered by the engineers. The rationally allocating the protection scheme of grid connection coupling point, which not only can judge the system fault in time, and judge it in DG island, and can still remain the protection features in the dynamic process of the island formation and in the operation mode of dividing island, can always have enough selectivity of the island fault is the problem to solve in this study. Through the selection of the grounding methods of DG grid connection transformer, the relationship between zero sequence current and zero sequence voltage is analyzed; the optimization of the protection scheme can inhibit DG interconnection and zero sequence over-voltage in network running-off, and quantity of zero sequence can be used as a stable criterion when the fault occurs, so as to work out a set of protection optimization schemes for DG interconnection transformer grounding system. Finally, through the COBASE system, a protection scheme of the wind power plant low-voltage grid is generated in this study, which verifies its excellent protection features in the DG connected to the grid system.
\end{abstract}

Keywords: Decoupling zero sequence protection; High Capacity for DG Interconnection; DG interconnection protection for wind power; interconnection trip; Auto-switch-in device based on frequency check,

\section{Introduction}

In the future, the construction of new power generation will be completed by the IPP (independent power producers). It is predicted that many disperse units will interconnect with smaller industrial and commercial facilities and power grids so as to reduce energy consumption of local load by the means of share or "peak shaving". Distributed generation DG needs the protection not only in the case of short circuit, but also in abnormal operating conditions ${ }^{[1,2,3]}$. Many abnormal conditions are applied to DG by the system. These abnormal operating conditions are as follows: over excitation, overvoltage, unbalanced current, abnormal frequency and shaft torque damage caused when the system conducts automatic reclosing. When subjected to such operating conditions, within a few seconds, DG damage or generator complete failures will occur. DG damage is one of the issues concerned by the owners of DG. On the other hand, for the power supply system, what is the widely concerned about is the DG access will lead to damage to their equipment or damage to their customers' equipment.

Most of the DG is usually connected to the distribution grid of power supply system and substations. These systems are designed in radiant configuration. DG island operation mode with external load is not allowed, there are two main reasons: 1. DG island operation can make the automatic reclosing, and the synchronization of generators/load 
(island) to the power supply system in manual switch complicated. 2. Power quality (voltage and frequency, and harmonic) usually cannot be maintained at an acceptable level in DG island operation, which will cause damage to the customers' equipment.

The well-designed protection should protect the interests of the DG owners and the power supply system, and obtain the utility with the lowest cost as much as possible. The main interconnection protection function is realized through the detection of DG asynchronous preventing system island operation, in other words, it is needed to determine whether it is off-grid or not ${ }^{[4,5,6]}$. The detection and tripping operation must be fast enough to allow the implementation of the automatic reclosing of the system ${ }^{[7]}$.

\section{Wind Field Protection Configuration Scheme based on Low Resistance Grounding}

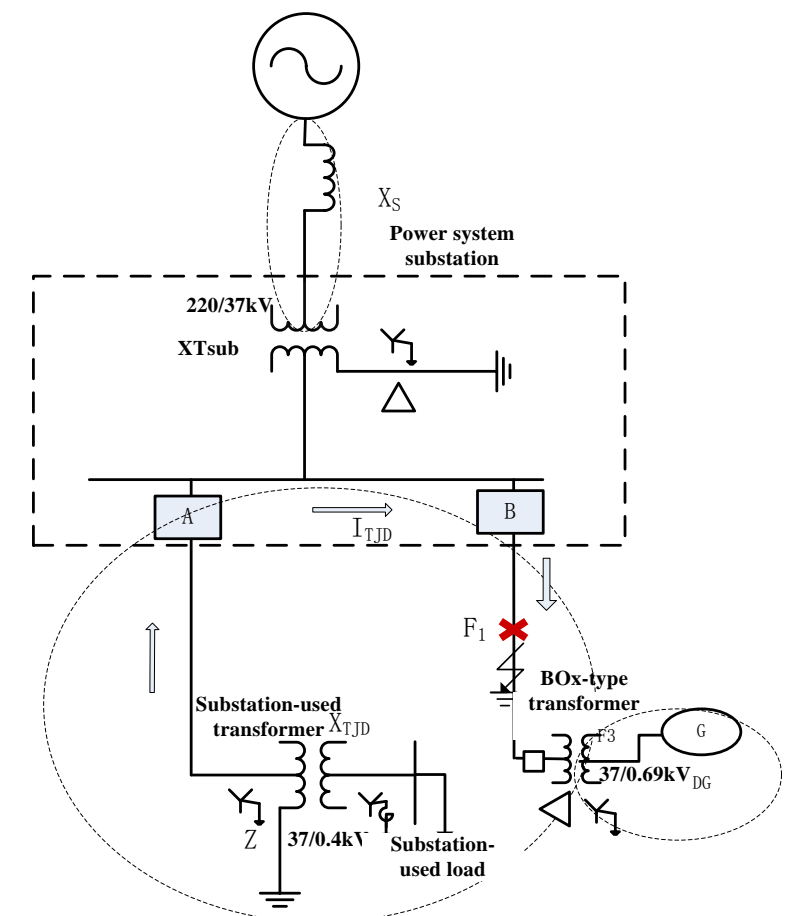

Figure 1. Wiring Diagram of DG Access to 110kV Substation

Figure 1 shows a schematic diagram of the transformer grounding mode of an interconnected wind power plant and related zero sequence protection principle. The total installed capacity of the wind field is 300 thousand $\mathrm{KW}$, and the fans are two types: $1.8 \mathrm{MW}$ and $2.3 \mathrm{MW}$, and their models are EN21 / EN23B. the voltage rating of interconnection is $220 \mathrm{kV}$. The wind field uses the low resistance grounding mode of grounding transformer, the parameters are shown in the following Table:

Table 1.

\begin{tabular}{cc|cc}
\hline $\begin{array}{c}\text { Rated } \\
\text { capacity }\end{array}$ & $\begin{array}{c}450 / 35 \mathrm{kV} \\
\mathrm{A}\end{array}$ & $\begin{array}{c}\text { Model } \\
\text { specificatio } \\
\text { ns }\end{array}$ & $\begin{array}{c}\text { DKSK-450- } \\
35\end{array}$ \\
\hline $\begin{array}{c}\text { Rated } \\
\text { voltage }\end{array}$ & $\begin{array}{c}36.75 / 0.4 \mathrm{k} \\
\mathrm{V}\end{array}$ & $\begin{array}{c}\text { Rated } \\
\text { current }\end{array}$ & $7.1 / 49.2 \mathrm{~A}$ \\
\hline $\begin{array}{c}\text { Impedance } \\
\text { voltage }\end{array}$ & $8 \%$ & $\begin{array}{c}\text { Connecting } \\
\text { group }\end{array}$ & Znyn11 \\
\hline
\end{tabular}


The grounding mode of $220 \mathrm{kV}$ transformer in the system station and in the wind interconnection field divides zero sequence network of the whole interconnection system into 3 separate systems. In which, the wind turbine grounding mode of the $0.4 \mathrm{kV}$ level is TN-C-S connection, namely, the PEN is divided into PE and N 3-phase 4-wire system. The box fan is connected through the cabinet type transformer, its connecting group of the transformer is D, yn11 wiring, and therefore, it will not provide zero-sequence short-circuit current for the wind station, its grounding protection is $0.35 \mathrm{In}, 0.2 \mathrm{~S}$, some independence without cooperation. The second independent system is the wind field system, the ground current size is determined by ground zero sequence impedance in the station, has nothing to do with the operation mode of DG. At the same time, it should take into account the grounding transformer tripping will cause the loss of low voltage side grounding, and overvoltage of the un-grounding system caused by the DG. Therefore, the tripping strategy adopted by the zero sequence current protection in the substation is: the time-limit I is set to trip the low voltage side section; the the time-limit II is set to trip the both sides circuit breakeres of the grounding transformer .

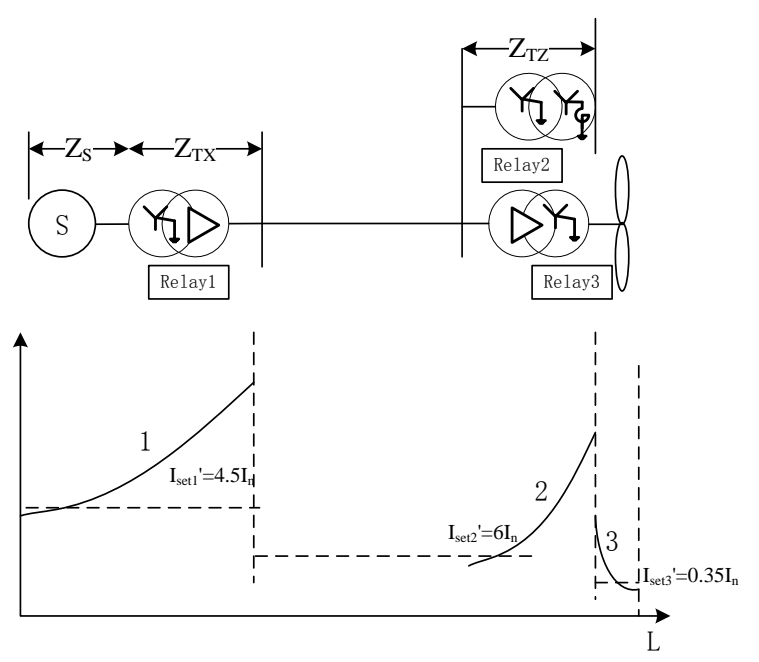

Figure 2. Zero Sequence Over Current Protection

Figure 2 reflects the zero-sequence current variation curve of the interconnection system in the wind field and the corresponding zero-sequence current protection setting configuration. It can be seen from the figure that, because of the different grounding methods, the whole zero-sequence system is divided into three independent zero sequence protection systems, namely $220 \mathrm{kV}, 35 \mathrm{kV}$ and $0.4 \mathrm{kV}$; for each system, the variation of its zero sequence current is continuous, while the curve in the three sections is sectional curve. Therefore, the three systems do not need to cooperate with each other, which can form an independent protection to act as the main and backup protections of the system. Because of the above characteristics, the time-limit I section set still can select the lower value, and the value given is based on the rated current of the itself voltage side. The use of zero sequence protection may not affect the normal DG operation mode, or it in or off grid operation mode shall not affect the protection set; the set mainly depends on the grounding model of the transformer and zero sequence impedance. The protection system formed is the protection configuration required by the system. Based on this idea, and the DG transition mode protection scheme of the zero sequence system is introduced, the specific scheme is as follows. 


\section{Implementation of the Protection Scheme Based on the DG Character and Breakpoints}

3.1.Preliminary Scheme for Protection and Separation Based on the Operation Mode

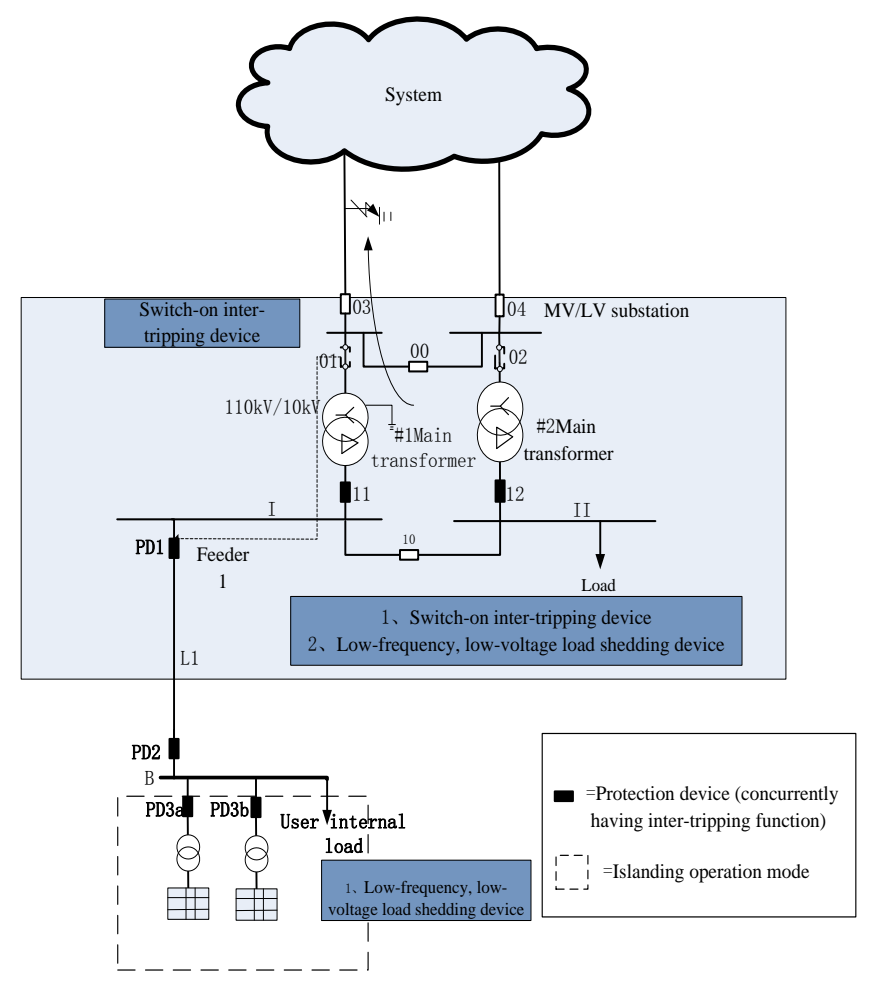

Figure 3. Primary System Diagram for the DG Connection to $110 \mathrm{kV}$ Substation

In this scheme, due to change of DG character, the high voltage side of grid-connected transformer is grounded, the opposite side line protection range of the system, the distance and zero sequence Section I, is extended into the transformer, and now is changed into $85 \%$ of line. Zero sequence protection on the high-voltage side of this transformer is used as main line protection, to trip off high-voltage side and bridge switch, to disconnect grid-connected line breaker at the same time. The breakpoint and incomplete protection configuration decided by the new operation mode is as follows: 


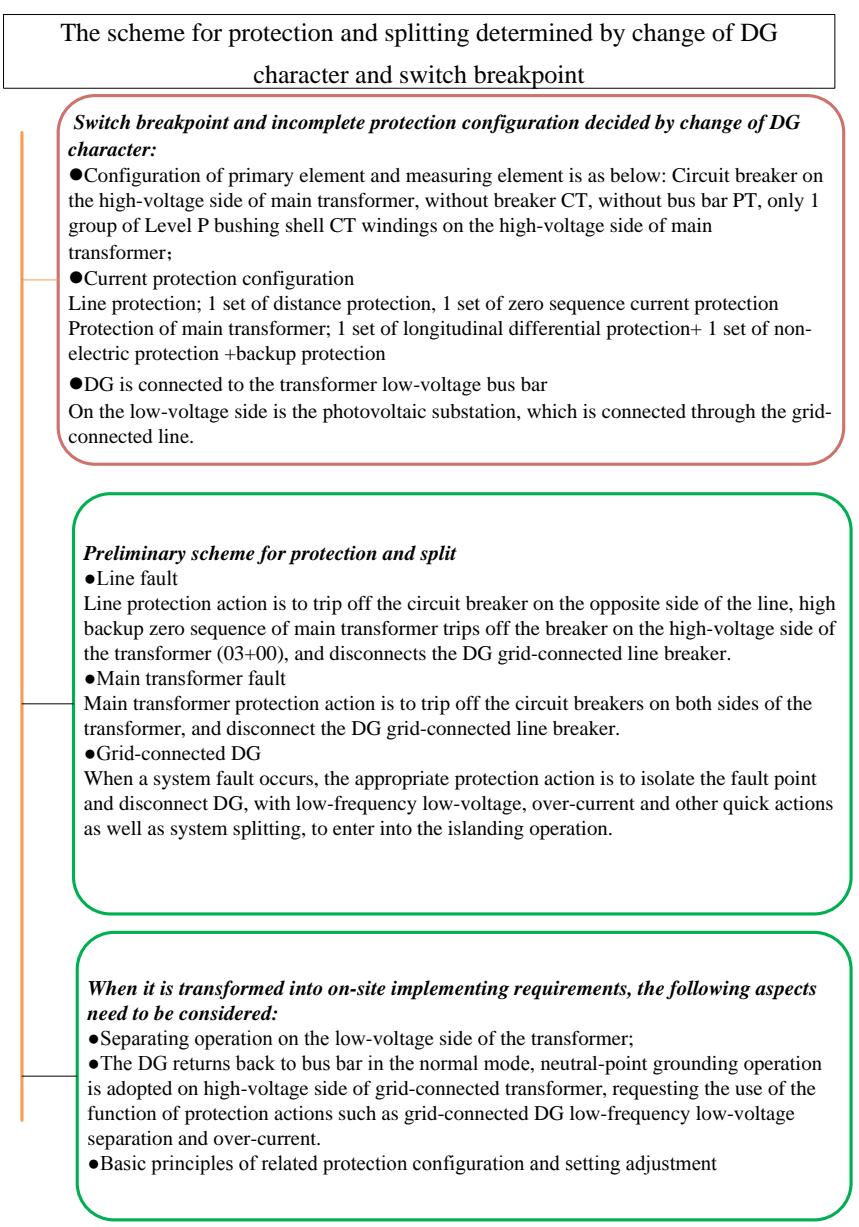

Figure 4. The Scheme for Protection and Splitting Determined by Change of DG Character and Switch Breakpoint

\subsection{Scheme for Breakpoint and Incomplete Protection Configuration Determined by the Transitional Operation Mode}

Figure3 shows the primary system diagram for the DG connection to $110 \mathrm{kV}$ substation, wherein the inner bridge connection is used for the MV/LV substation, No. 01, and 02 (virtual breakers, drawn in dotted line in fig 3, 01 equals to 00+03), and the functions of inter-tripping gird-connected line L1 are realized through the inter-tripping function of No. 03 circuit breaker. Due to DG small size in the initial design, DG grid-connected substation is considered as the terminal substation without small power source. Therefore, breakers of substation $(03,04)$ are not configured with protection devices, and the first choice is to use the \#1 and \#2 transformer high voltage side backup protections. Generally speaking, as long as the requirement of protection sensitivity is satisfied, high voltage side of the transformer is not configured with distance protection. At the same time, photovoltaic power has a poor ability to supply short-circuit current when a system fault occurs, and so far there is no algorithm which can be applied to the engineering practice, so fault source is required to reliably and accurately reflect system fault. Based on the above two factors, the most direct solution is to consider the DG as "Small Power Source", to ensure 110kV side grounding of substation. Meanwhile, zero sequence overcurrent protection in high voltage side backup protection is deemed as No. 03, 04 line protection of load side, and the setting principle is also to follow the principle of line protection configuration. Section I is set to avoid the fault at the end of the line; Section II, protect the whole line and ensure its sensitivity, and cooperate with all 
outgoing lines of $220 \mathrm{kV}$ substation on the opposite side. Thus, when a fault occurs to the system, enough zero sequence fault current is provided from high-voltage side grounding point of the transformer to fault point, to ensure high backup protection action of the transformer, and No. 03,( or 04) breaker trip off. The analysis show that the determination of which transformer is grounded is up to from which one the DG is interconnected. Grounding point is to provide zero sequence current when a system fault occurs, and this zero sequence system has nothing to do with the DG. However, the DG is interconnected in this main transformer, after the breakers (03 and 00) on the high-voltage side of this transformer trips off, the DG will be effectively isolated from the grid.

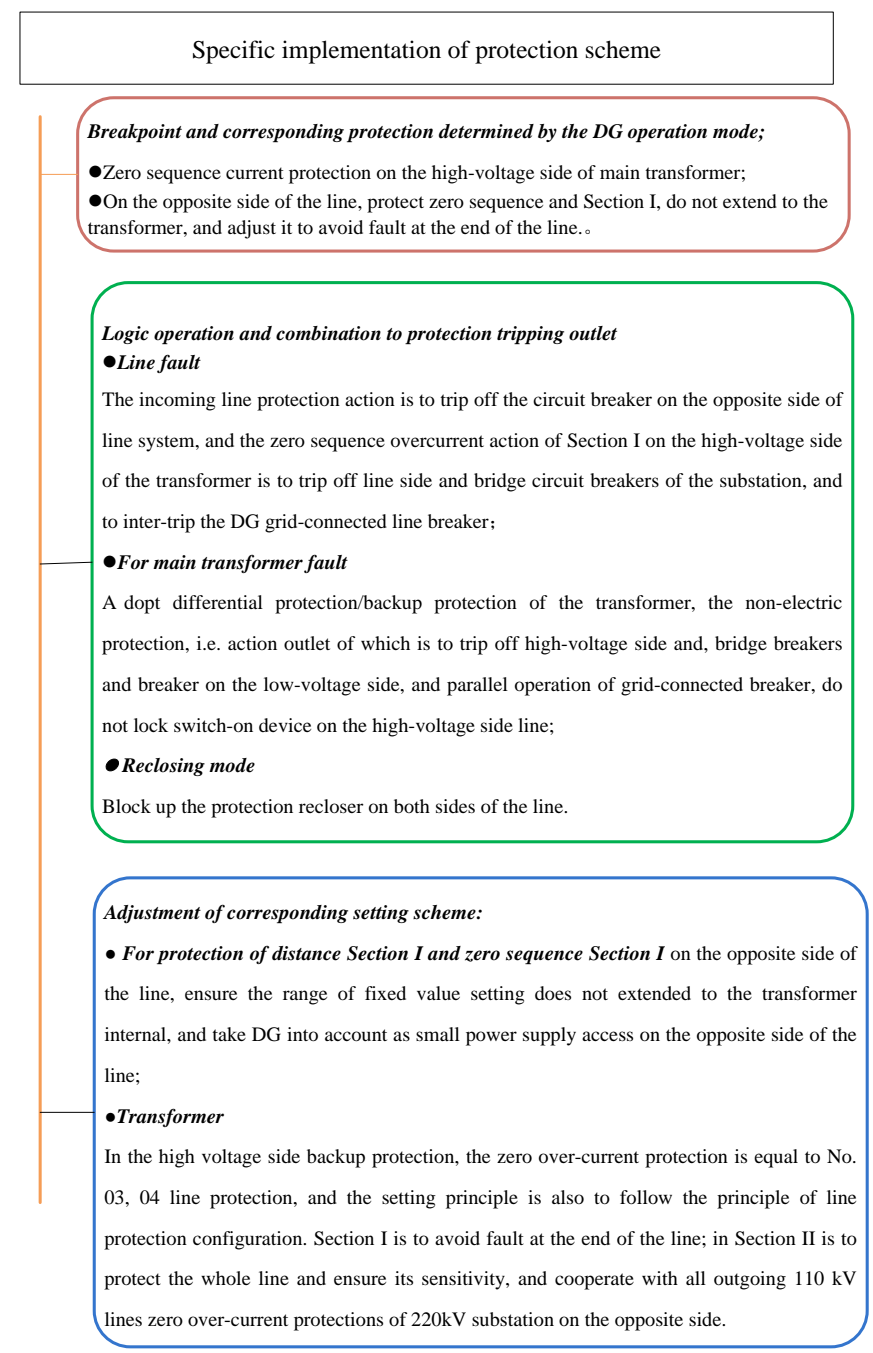

Figure 5. Specific Implementation of Protection Scheme

\subsection{Fault Response Analysis}

Based on current protection configuration, protect action is as follows: Wen line fault occurs, breakers on both ends trip off but do not re-close, switch-on device action, disconnect the DG grid-connected line. At this point, local area enters the islanding operation. The low-voltage side back-up protection, the time-limit protection, being used as the main protection of bus bar on the low-voltage side operate, trip off the transformer breaker, disconnect the DG grid-connected line, and lock sectionalized switch-on devices on the low-voltage side. As for differential and non-electric protection 
action of main transformer, do not lock high voltage side incoming lines and the low voltage side sectionalized switch-on devices.

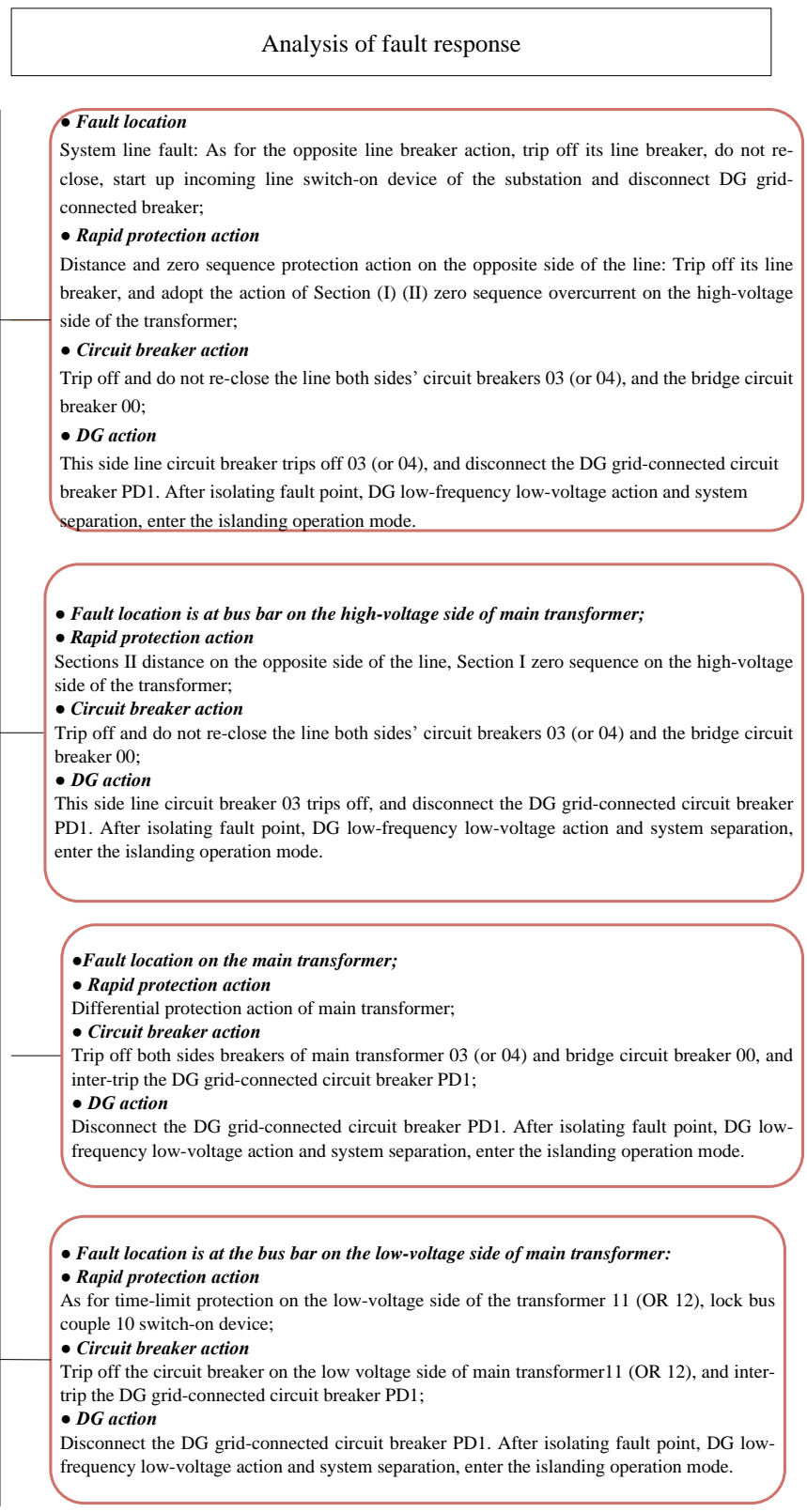

\section{Figure 6. Analysis of Fault Response}

When one transformer runs, if a fault occurs to line of the system, through No. 3 breaker action, directly cut off the connected channel of photovoltaic and the system. Voltage loss of the bus bar on the high voltage side does not affect normal action of switch-on device, but there are effects when in two transformers simultaneous running mode. No. 3 breaker action on the high voltage side of the transformer cannot completely cut off the DG-system connection channel, thus, it will affect the success rate of switch-on device. Therefore, in the high backup protection action, it is required to disconnect photovoltaic grid-connected line L1 of this substation, which can be satisfied according to current protection configuration. Meanwhile, when the original parallel operation of two transformers is changed into the operation of one transformer, low-frequency low-voltage device on the low-voltage side is required to balance the corresponding load, to ensure the quality of power supply. After balancing, the problem 
of tripped-off load can be solved with the help of the hand in hand of distribution network.

It can be seen from the protection range of transformer high voltage side backup zero sequence protection, the protection range of No. 03 breaker is extended to bus bar on the high-voltage side. The analysis shows that when a fault occurs to the bus bar on the high-voltage side, if there is no effective measure of identification and locking, switch-on device will act again and recluse to the faulty bus bar, impacting the system again. The best solution is to install the bus bar differential protection on the high-voltage side of the DG grid-connected substation. When a fault occurs to bus bar, output the locking switch-on device through action nodes of bus bar differential protection. Without configuration of bus bar differential protection, compare the magnitude of fault current of No. 03, 01 (the virtual breaker, drawn in dotted line in fig 3 equal to 00+03) to judge whether a fault occurs to bus bar. If I03 > I01, it means that a fault occurs to bus bar. Only when fault points are located between two switches, short-circuit current of No. 03 breaker provided by the system is much more than that of No. 01 one provided by the DG. It is important to note that zero sequence current is not a basis for judgment. The basis for judgment is to directly compare the magnitude of short circuit current provided by power source on both sides.

\section{Analysis of Project Case}

Figure 7 shows the primary wiring diagram of a wind power plant, its capacity is $300000 \mathrm{kw}$, the fans are two types of $1.8 \mathrm{MW}$ and $2.3 \mathrm{MW}$, and the models are respectively EN21 / EN23B. The voltage level of grid connection is $220 \mathrm{kV}$. Its protection configuration is basically the same as the conventional substation, and the fans are configured with the anti-island device. Considering the nature of doubly-fed machine in the wind power plant, its step splitting device is out of operation.Combining with the characteristics of low resistance grounding on the low voltage side, this study made a detailed analysis of the grounding transformer protection configuration.

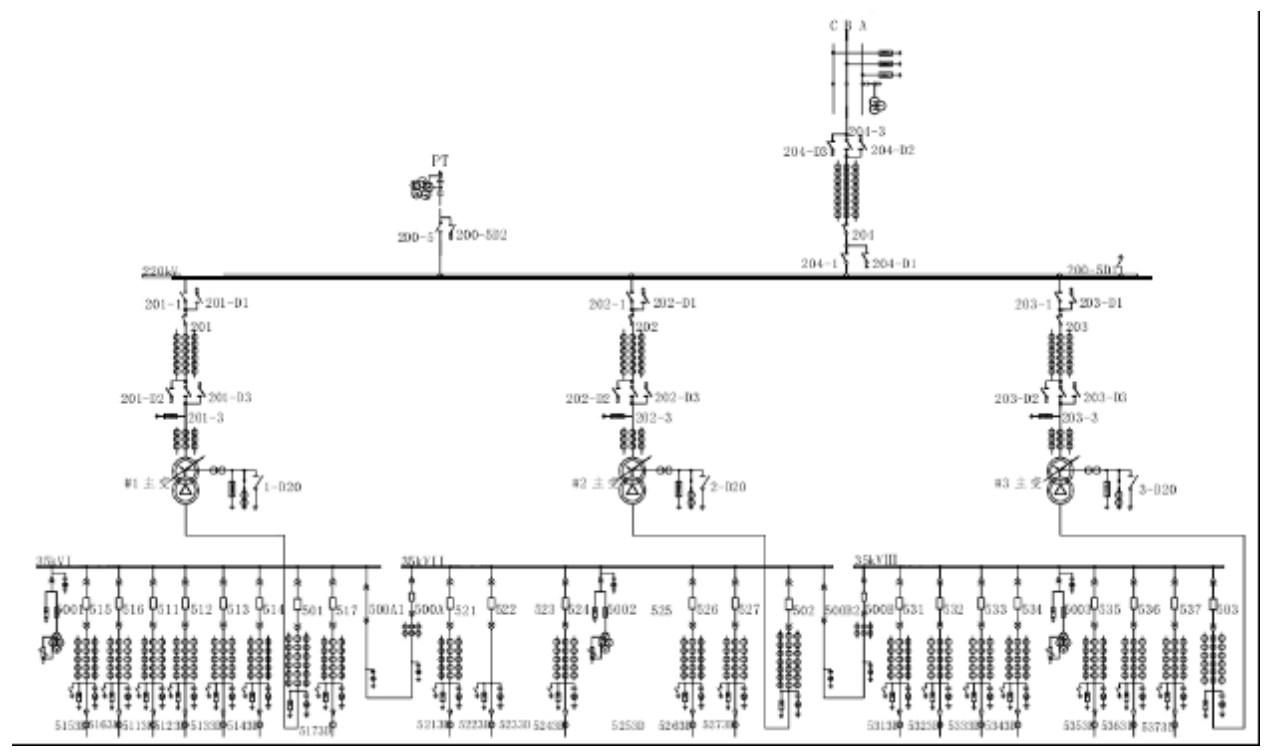

Figure 7. Primary Wiring Diagram of a Wind Power Plant

According to the latest national standard GB/T 50064-2014 standard of "Over Voltage Protection and Insulation Coordination of AC Electrical Devices ", in which, Clause 3.1.4 provides: $6 \mathrm{kV} \sim 35 \mathrm{kV}$ is mainly composed of the distribution system of cable line, the power system of power plant, the collector system of wind power plant and the power 
supply system of industrial enterprises except the mines. When the single-phase grounding fault capacitive current is larger, the neutral low resistance grounding mode can be used. Under the premise of meeting the single-phase grounding relay protection reliability and overvoltage and insulation coordination, the resistor of neutral point resistance of the transformer should chooses a larger value. The model of the three main transformers of the wind power plant is SZ11-100000/220, its connection mode is $Y / \triangle$ connection mode, and it is connected with grid with the single busbar connection mode. The low voltage side is grounded in the form of grounding transformer with resistors. The parameters of the grounding transformer are as follows: the model of DKSK-450/35, the capacity of $450 \mathrm{kva}$, the rated current of $7.1 \mathrm{~A}$, the rated voltage of $36.75 \mathrm{KV}$, and the grounding resistance of $108 \mathrm{ohm}$.

Because it is grounded through low-resistance, when the single-phase grounding fault occurs, the tripping will occur too. It is calculated that the zero-sequence current is 54A when the grounding transformer fault occurs. Zero-sequence current Phase I is taken as 44A after considering 1.2-times zero-sequence factors, supposing the time is after the collector lines action, the time is taken as $0.6 \mathrm{~S}$, then it trippes the low voltage side sectional circuit breaker; Zero-sequence protection II stage is taken as $22 \mathrm{~A}$ after considering it is coordinated with the set wire stage II, the time is taken as $1.8 \mathrm{~S}$, then it trips the circuit breaker at the grounding transformer high voltage side and the circuit breaker at the low voltage side of power transformer; the two-stage current protection is configured as follows: Stage I avoids the maximum short-circuit current at the transformer low voltage side and the transformer inrush current, is taken as $240 \mathrm{~A}$, the time is $\mathrm{OS}$, the grounding transformer in-line circuit breaker trips; In the second stage, the sensitivity of the low-voltage side is not less than 1.5 , which is taken as $40 \mathrm{~A}$; and the time is consistent with the time of the breaker at the low-voltage side circuit of the backup protection of the power supply transformer. The circuit breaker of the high voltage side of the grounding transformer and the circuit breaker at the low voltage side of power supply transformer are tripped.

Figure 8 is the requirements for the low voltage ride-through in The Typical Design of Electric Power System in Wind Power Plant. It can be seen from the figure that, the low voltage ride-through requirements are as follows: when the voltage at the network of the wind power plant drops to $20 \%$ nominal voltage, the wind turbine can ensure the continuous operation of the network for $625 \mathrm{~ms}$. When the voltage at the network of the wind power plant can be recovered to $90 \%$ of the nominal voltage within $2 \mathrm{~S}$ after the occurrence of the network drop, and the wind turbine generator in the wind power can ensure the continuous operation of the network.

When the system fault occurs, the low voltage ride through time required by the wind power is $2 \mathrm{~S}$, so the $2.3 \mathrm{~S}$ action time of ride- through stage II can avoid this time and ensure no malfunction when the system fault occurs, therefore, the protection at this section without the requirements for the direction, in this way can it be used as backup protection of the system.

At the same time, the grounding transformer tripping will enable low-voltage side lose the grounding is taken into account, despite the high side is configured with the gap over-voltage protection, it is also needed to consider the circuit breaker at the low voltage side of the tripping power transformer, so as to prevent over- voltage from transferring to the high voltage side. 


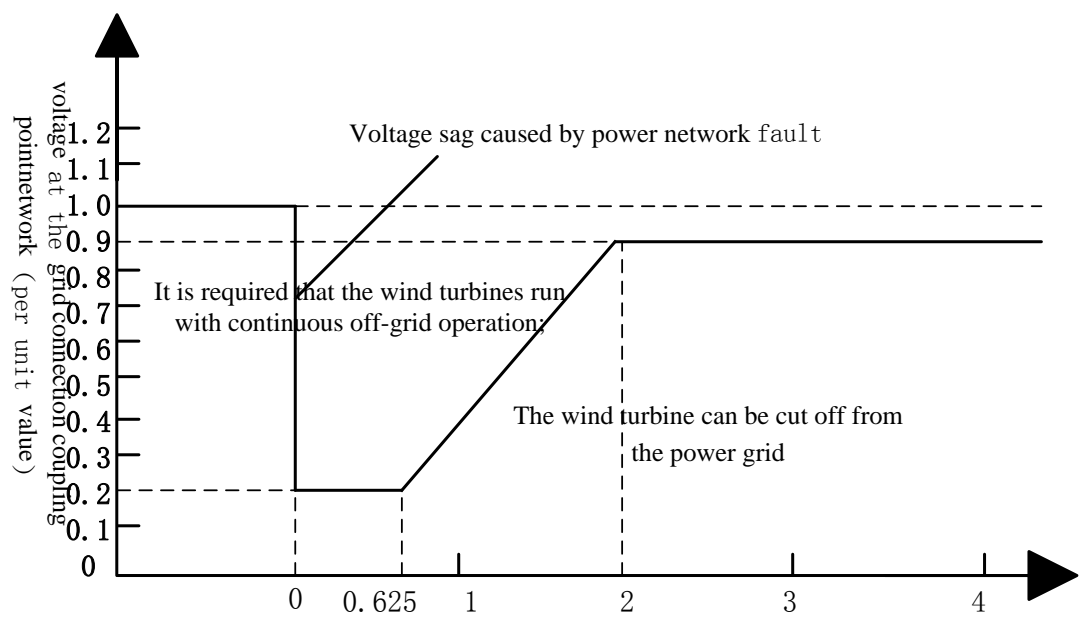

Figure 8. Schematic Diagram of Low-voltage Ride-through at the System Fault of the Wind Power Station

\section{Conclusions}

This paper introduces the Coordinated and Comprehensive Protection Scheme for the Transitional Period with increasing DG incorporation. With the increase of DG capacity, the level of short-circuit current increases, DG character is also changed from "Pure Load" into "Small Power Source", and the existing protection and breakpoint have been unable to meet the needs of transition. Under the conditions of existing protection and breakpoint, through continuously matching with primary system operation mode, the scheme is constantly optimized, and information and state variables are continuously exchanged, to form the protective control interface adapted to the one-time system, to achieve a dual purpose, namely, safe operation of the system and continuous power supply. First of all, within the range of fault isolation, determine the new boundary to collect switch breakpoints, and give an overall consideration to whether system separation and islanding operation run steadily after the boundary point is determined. By changing the grounding mode of the transformer, system fault can be effectively found. At the same time, with the aid of zero sequence overcurrent and breaker on the high voltage side of the transformer, it is ensured that effective isolation of system fault can be achieved. Through setting coordination between protection device and automatic device, it is ensured that after fault isolation, load can be effectively transferred. In the DG islanding operation, the DG can ensure continuous power supply for local load, to realize the micro-grid protective function. This scheme provides the effective means to solve the problem that continuous DG connection to the grid system.

\section{References}

[1] BRAHMA S. Development of an adaptive protection scheme for power distribution systems with high penetration of distributed generation [D]. Clemson, SC , USA : Clemson University ,2003.

[2] PERERAN , RAJAPA KSE A D. Agent-based protection scheme for distribution networks with distributed generators/ /Proceedings of Power Engineering Society General Meeting ,June 18-22, 2006 , Mont real, Canada : 3793-3798.

[3] DING Lei, PAN Zhencun, WANG Bin. Impact on reliability of distribution networks with dispersed generators. Automation of Electric Power Systems, 2007, 31 (20) : 89-93.

[4] HUANG Wei, L EI Jinyong, XIA Xiang, et al. Influence of distributed generation on phase2to2phase short circuit protection in distribution network. Automation of Electric Power Systems ,2008, 32 (1) : 93-97.

[5] ZHANG Qingjie, LU Yuping. A new dist ributed protection principle based on adaptive division for relevant fault area.Automation of Electric Power Systems , 2008 , 32 (7) : 39-43. 
[6] GIRGIS A , BRAHMA S. Effect of distributed generation on protective device coordination in distribution system/ /Proceedings of 2001 Large Engineering Systems Conference on Power Engineering, Jul 11-13, 2001, Halifax , Canada. Piscataway, NJ , USA : IEEE , 2001 : 115-119.

[7] TANG Haijun. Research on feeder automation model of distribution network GPRS 2 based. Automation of Electric Power Systems,2006,30(7):104 -106.

[8] WANG Shouxiang,LI Xiaojing, XIAO Zhaoxia,et al. Multi 2 agent approach for service restoration of distribution system containing distributed generation. Automation of Electric Power Systems,2007,31(10):61- 65 .

[9] LIN Xia , LU Yuping, WANG Lianhe. New current protection scheme considering distributed generation impact .Automation of Electric Power Systems, 2008 , 32 (20) : 50-56.

[10] WEI Zhinong, HE Hua, ZHENG Yuping. A novel algorithm for fault location in power distribution network. Automation of Electric Power Systems,2001,25(14):48 -50.

[11] Ropp M, Begovic M, Rohatgi A, et al. Determining the relative effectiveness of islanding detection methods using phase criteria and nondetection zones[J]. IEEE Transactions on Energy Conversion, 2000, 15 (3): 290-296.

[12] Sun H L, Lopes L, Luo Z X. Analysis and comparison of islanding detection methods using a new load parameter space[C]. 30th IEEE Industrial Electronics Society Conference, Korea, 2004: 1172-1177.

[13] Smith G A, Onions P A, Infield D G. Predicting islanding operation of grid connected PV inverters[J]. IEE Proceedings on Electric Power Applications, 2000, 147 (1): 1-6.

[14] Hannu Jaakko Laaksonen.Protection Principles for Future Microgrids [J]. IEEE Transactions on power systems,2010,25(12)

[15] Zhang Hao, Zhu Shouzhen, Study on grounding project of distributed generation connecting electric grid[J]. Journal of Shenyang Institute of Engineering(N atural Science), 2006, 2(3)

[16] Zhang Yanxia,Dai Fengxian.New Schemes of Feeder Protection for Distribution Networks Including Distributed Generation[J]. Automation of Electric Power Systems,2009,33(12)

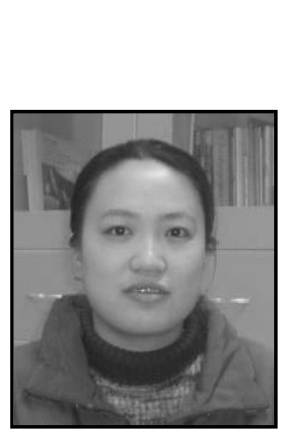

\section{Authors}

Xia Lin, female, Doctorate, associate advanced engineer, carries out the research on protection and control of the distributed power generation system, field engineering application and so on . 
International Journal of Grid and Distributed Computing Vol. 9, No. 8 (2016) 